\title{
Acute Myeloid Leukemia in Remission
}

National Cancer Institute

\section{Source}

National Cancer Institute. Acute Myeloid Leukemia in Remission. NCI Thesaurus. Code C3588.

Acute myeloid leukemia not growing, responding to treatment. 